\title{
Neuropathic Arthropathy of The Hand Joints in Patient with Arnold-Chiari Malformation Type I And Syringomyelia Caused by Cerebellopontine Angle Arachnoid Cyst: A Case Report
}

\author{
Yahya $^{1}$, Muhammad Saiful Ardhi ${ }^{2}$, Fadil $^{2}$ \\ ${ }^{1}$ Resident of Neurology Department Medical Faculty of Airlangga University/dr. Soetomo General \\ Hospital Surabaya \\ ${ }^{2}$ Lecturer of Neurology Department Medical Faculty of Airlangga University/dr. \\ Soetomo General Hospital Surabaya
}

\begin{abstract}
:
Introduction :

Neuropathic arthropathy (NA), is a progressive degenerative arthropathy associated with an underlying central or peripheral neurologic disorder. Neuropathic arthropathy has been observed in a variety of conditions including syringomyelia, diabetes mellitus, and peripheral nerve disorders. The shoulder and elbow joints are the most frequently affected while the hand joints are quite rarely seen in the cases of NA caused by syringomyelia. In this article, we report the case of neuropathic arthropathy in a patient with Arnold-Chiari malformation type I and syringomyelia probably caused by a cerebellopontine angle (CPA) arachnoid cyst.
\end{abstract}

\section{Case}

A 39-years old man came with slight weakness in both upper and lower extremities and spastic-related deformities in both hands accompanied by a history of headache. Cranial nerves examinations showed rotator nystagmus. Plain radiograph examinations of both hands demonstrated destructive osteoarthritic changes and multiple ossified intraarticular bodies consistent with intra-articular osteophytes. He was investigated with magnetic resonance imaging (MRI) of the brain and the cervicothoracic spine and they revealed arachnoid cyst at cerebellopontine cisterna causing the cerebellar tonsils downward displacement across foramen magnum (Arnold-Chiari type I malformation) with communican hydrocephalus and a syringomyelic cavity extending from $\mathrm{C} 2$ to $\mathrm{Th} 5$ vertebral levels. Bed rest and lowering-intracranial pressure therapy with acetazolamide were given to the patient.

\section{Conclusion}

Development of neuropathic atrophy following the diagnosis and surgery of syringomyelia should be considered especially in destructive cases without evident pain complaint and the underlying neurological problem should be surmounted. 
Keywords: neuropathic atrophy, Arnold-Chiari malformation, syringomyelia.

\section{Introduction}

Neuropathic arthropathy is a progressive degenerative arthropathy associated with underlying central or peripheral nervous disorders (Aly et al. 2013). This disorder was first discovered by Charcot in 1868 and often found in patients with syringomyelia $(25 \%)$, diabetes mellitus $(0.16 \%-2.5 \%)$, and other peripheral nerve disorders such as tabes dorsalis (5\% - 10\%) (Aly et al. 2013; Choudhury et al. 2019; Yanı, Tuncer, and Seçkin 2004). Imaging results in neuropathic arthropathy cases referred as "6D", including dense bones (subchondral sclerosis), destruction (damage) to the articular cartilage, disorganization (joint deformity), debris (splinter), distension (fluid), and dislocation. Shoulder and elbow joints are the most frequently affected sites in patients with syringomyelia. Some cases show involvement of hand joints due to syringomyelia (Nacir et al. 2010; Yanık et al. 2004).

Type I Arnold-Chiari malformation is a downward shift of the cerebellar tonsils through the foramen magnum into the cervical canal, caused by volume decrease of the posterior fossa. Various conditions such as skull base deformities, bony abnormalities, craniocerebral disproportion, and supra- or infratentorial tumors can cause caudal displacement of cerebellar tonsils with or without syringomyelia (Galarza, LópezGuerrero, and Martínez-Lage 2010; Martínez-Lage et al. 2008). Arachnoid cysts at the cerebellopontine angle are rarely asymptomatic. There are only a few case reports in which Arnold-Chiari type I malformation and syringomyelia are thought to be caused by arachnoid cysts due to tonsillar herniation (Joshi et al. 2013). Arachnoid cyst is a benign accumulation of cerebrospinal fluid, caused by duplication of the arachnoid membrane in the brain formation phase and can lead to several non-specific symptoms. To date, it is not known whether the Arnold-Chiari malformation and arachnoid cyst occur simultaneously or co-occur. Arnold-Chiari malformation and dense posterior fossa structures are the most common etiology of obstructed cerebrospinal fluid flow. It is suspected that arachnoid cysts play a role in tonsillar herniation and syringomyelia (Galarza et al. 2010). In this article, we report a case of neuropathic arthropathy of hand joints caused by cerebellopontine arachnoid cysts.

\section{Case Report}

A 39-year-old man, working as a driver, presented with slight weakness in both upper and lower extremities as well as spastic deformities in both hands and a history of headache. Bladder and digestive function were within normal limits, general physical examination did not reveal any abnormalities. Cranial nerves examination showed rotatoar nystagmus. Patient showed slight weakness in upper and lower extremities (4/4 muscle strength). On sensory examination, sensory disturbances were found in upper extremities. Patient has normal physiological reflexes and no pathological reflexes. Hematological and blood chemistry examinations were within normal limits. Chest Xray examination showed normal results, and radiographic examination of both hands 
showed osteoarthritis of PIP (proximal interphalangeal) joint, DIP (distal interphalangeal) joint of digit 2,3,4,5 right and left manus and MCP

(metacarpophalangeal) joint of digits 2, 4, 5 of left manus, bowing deformity of left 5th metacarpal (Fig. 1). The patient underwent magnetic resonance imaging (MRI) examination of brain and cervicothoracic vertebrae, and the arachnoid cyst was found in cerebellopontine cistern leads to a downward shift of cerebellar tonsils through the foramen magnum (Arnold-Chiari malformation type I) with communicating hydrocephalus (Fig. 2 ) and syringomyelia cavity at C2 to Th5 vertebrae (Fig. 3). Patient was managed by bed rest and reduction of intracranial pressure using acetazolamide. Patient refused to undergo sub-occipital decompression surgery.
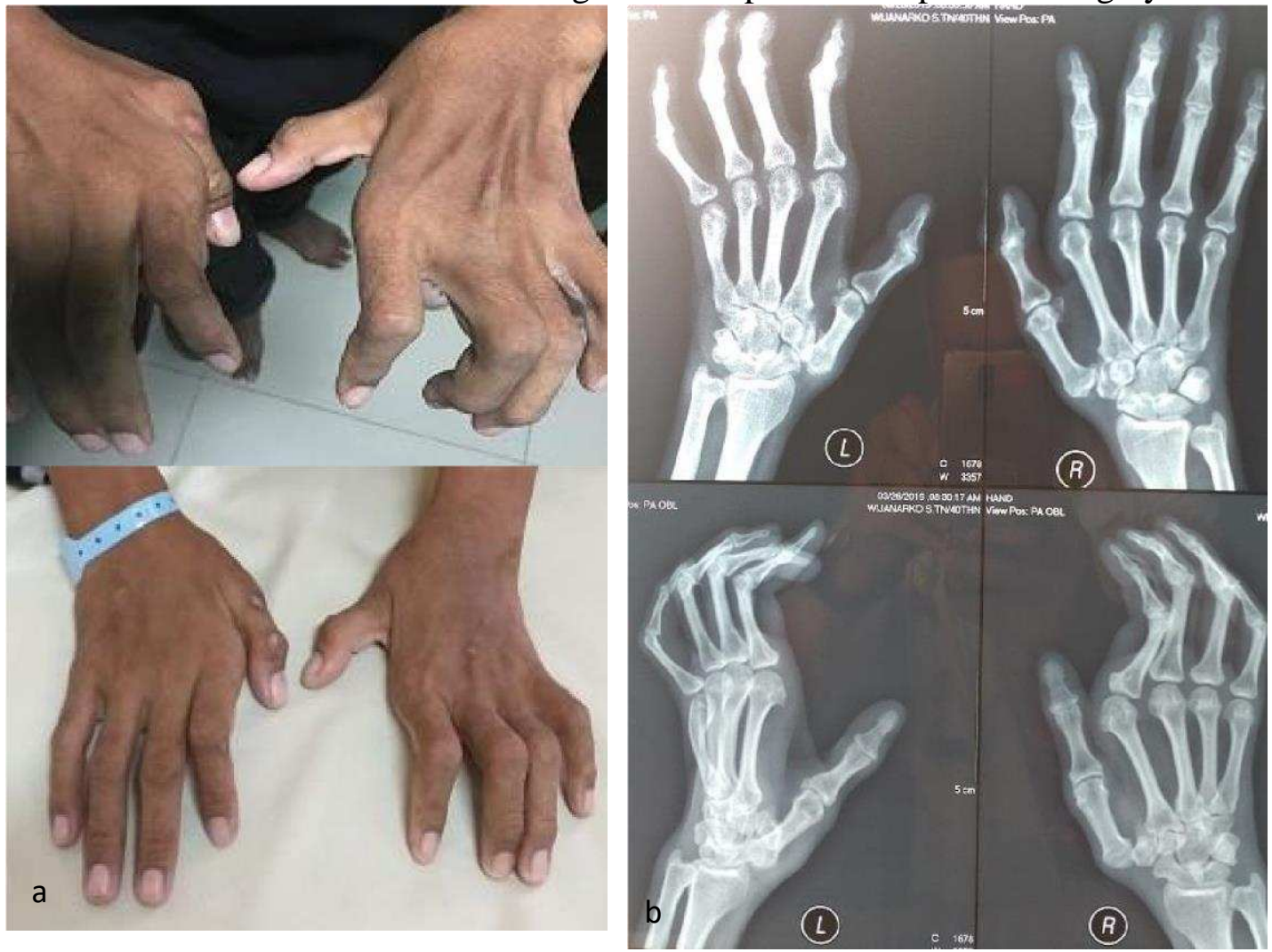

Figure 1. (a) Dorsal aspects of right and left hands showing flexion contractures of fingers. (b) Radiograph of both hands from anteroposterior side. Osteophytes were seen in proximal interphalangeal joints dan distal interphalangeal joints of second, third, fourth, and fifth of right and left hands; and in second, fourth, and fifth metacarpophalang joints of left hand with deformity at the fifth metacarpal joint of left hand. 

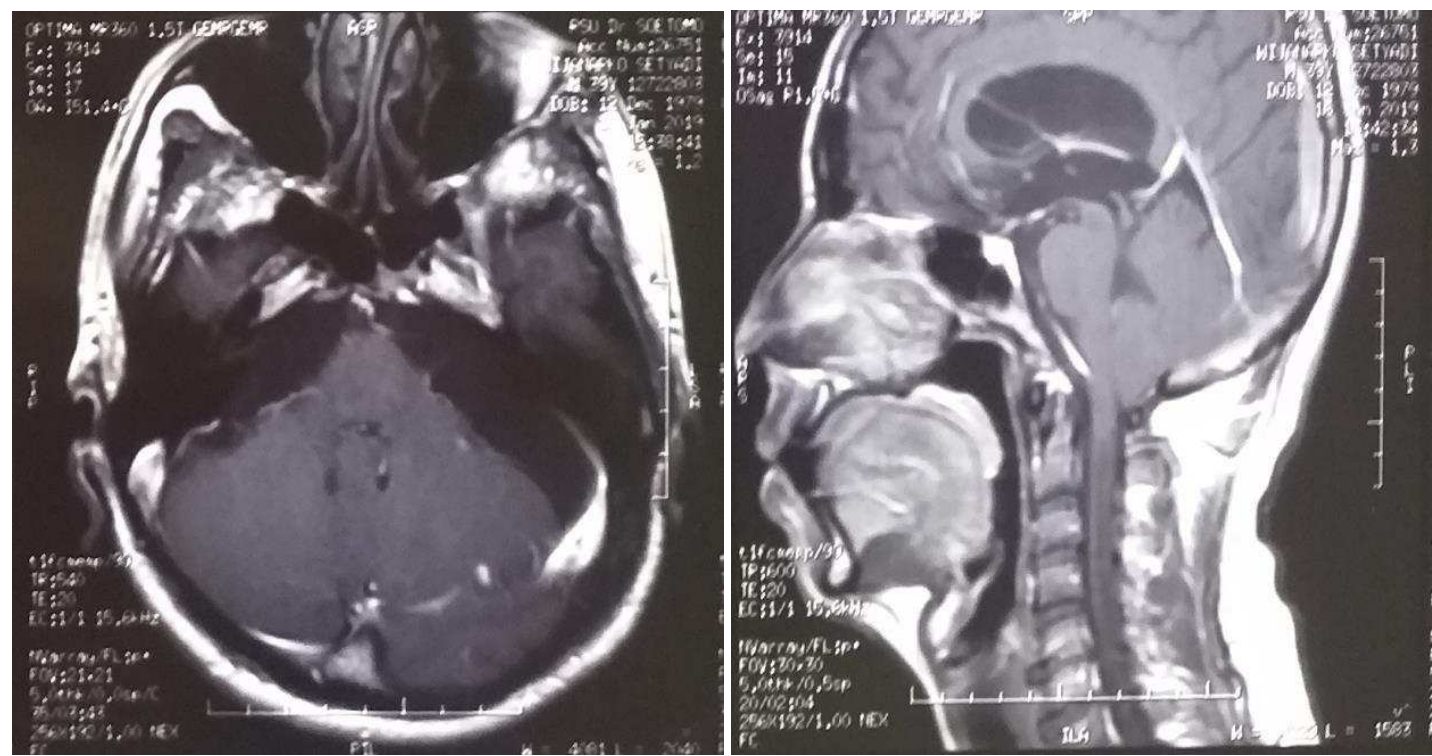

Figure 2. T1-weighted magnetic resonance imaging (MRI) without contrast showed (a) arachnoid cyst at cerebellopontine angle and (b) downward displacement of cerebellar tonsils with obstruction of the foramen magnum and communicating hydrocephalus.

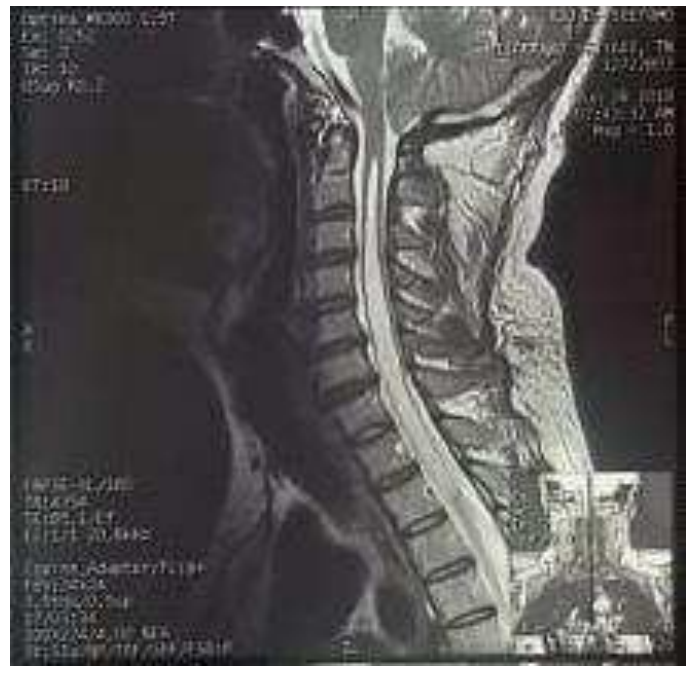

Figure 3. MRI imaging (T2-weighted sagittal) with contrast revealed syringomyelia cavity (hyperintense syrinx) from vertebral level C2 to Th5 with Type I Arnold-Chiari malformation.

\section{DISCUSSION}

Type I Arnold-Chiari malformation is downward displacement of cerebellar tonsils at least 3 to $5 \mathrm{~mm}$ below foramen magnum with or without syringomyelia (Martínez-Lage et al. 2008; Milhorat et al. 1999). It is one of four types of cerebellum malformations discovered by Hans Chiari in 1891. Most patients with type I 
ArnoldChiari malformation have clinical manifestations of posterior fossa or foramen magnum compression (headache and neck pain, cerebellum sign, cranial nerves dysfunction, dysphagia, sleep apnea, and breathing difficulties) or spinal cord-related disorders (dysesthesia, dissociative sensory disturbances, fine movement disorders, stiffness, scoliosis, or sphincter disturbances) (J F Martínez-Lage 1, M J Almagro, J Ros de San Pedro, A Ruiz-Espejo 2007).

Descending uprooting of cerebellar tonsils that blocks cerebrospinal liquid stream from and towards the spinal compartment in foramen magnum will bring about syringomyelia(Oldfield et al. 1994). Syringomyelia is an uncommon, persistent, moderate, and degenerative problem of spinal line. This problem is portrayed by the presence of a liquid filled longitudinal cavity (syrinx) in cervical and cervicothoracal districts. Syringomyelia is seen as in $75-85 \%$ of patients with type I Arnold-Chiari malformation(Nacir et al. 2010). Arachnoid sores were related with clinical and imaging discoveries of tonsillar herniation and syringomyelia in a few case reports (Galarza et al. 2010; J F Martínez-Lage 1, M J Almagro, J Ros de San Pedro, A RuizEspejo 2007; Martínez-Lage et al. 2008)(Al-Holou et al. 2013).

Arachnoid cyst, a benign accumulation of cerebrospinal fluid, can lead to abnormal arachnoid formation. Arachnoid cyst accounts for approximately $1 \%$ of intracranial mass lesions (Cincu, Agrawal, and Eiras 2007). Middle cranial fossa was the most frequently affected site $(60 \%)$, followed by cerebellopontine angle (10\%) and suprasellar region (9\%) (Cincu et al. 2007; Esra Gurkas1, Buket Yucel Altan2, Kivilcim Gucuyener3 2015; Ramesh S 2015). Arachnoid cysts at cerebellopontine angle resulted in volume decrease of posterior cranial fossa, which is the main etiology of type I Arnold-Chiari malformation. In most cases, arachnoid cysts are precipitating factors that pushed cerebellar tonsils downward through the foramen magnum (Galarza et al. 2010), whereas several other cases reported tonsillar herniation and syringomyelia in various conditions, such as supra- and infratentorial tumors, brain pseudotumours, craniosynostosis, cranio-encephalic disproportion, excessive cerebrospinal fluid drainage, or cerebrospinal fluid drainage in vertebrae (Arunkumar, Korah, and Chandy 1998; Galarza et al. 2010).

Cerebelopontine angle arachnoid cysts are usually asymptomatic, however, they may enlarge and result in signs and symptoms due to pressure on the area of innervation or from increased intracranial pressure. The main clinical symptoms of cerebellopontine angle arachnoid cyst are headache and ataxia. Patients also complain of symptoms such as nausea, vomiting, cerebellar signs and symptoms, changes in character, and impaired memory(Esra Gurkas1, Buket Yucel Altan2, Kivilcim Gucuyener3 2015). These cysts may also lead to dysfunction of various specific cranial nerves such as II, IV, VI nerves (causing diplopia), V (causing trigeminal neuralgia), VII (causing congenital or acquired facial paralysis), VIII (causing hearing loss, tinnitus, and vertigo), IX (glossopharyngeal neuralgia), and $\mathrm{X}$ nerves (causing hoarseness and dysphagia) (Esra Gurkas1, Buket Yucel Altan2, Kivilcim Gucuyener3 2015; Gönül E, Izci Y 2007; Hayden et al. 2007). 
Cases reported by Martinez et al (2007) and Bauer et al (2005) is similar to our case, in which obstruction of cerebrospinal fluid flow through the foramen magnum was caused by an acquired Arnold-Chiari malformation that seemed to be derived from displacement of the cerebellum due to compression by the cerebellopontine angle arachnoid cyst. As seen in our patient, arachnoid cyst resulted in hydrocephalus(Bauer, Mueller, and Oró 2005; Cincu et al. 2007). Hydrocephalus may also be a contributing factor, but it appears that tonsillar herniation and syringomyelia in this patient are due to pressure from cerebellopontine angle arachnoid cyst. Initial therapy for hydrocephalus in this patient aims to relieve increased intracranial pressure to prevent the risk of acute cerebellar herniation that may occur during procedures in posterior fossa (J F Martínez-Lage 1, M J Almagro, J Ros de San Pedro, A Ruiz-Espejo 2007).

Neuropathic arthropathy has been reported in many cases of syringomyelia and was initially discovered by Sokoloff. Neuropathic arthropathy may occur in the early or late stages of syringomyelia. Involvement of shoulder and elbows joints are more common in cases of neuropathic arthropathy associated with syringomyelia. However, hands involvement is rare. The pathogenesis of neuropathic arthropathy is still a controversial topic. Loss of nociceptive stimulation and trauma can result in neuropathic arthropathy in extremities. Changes in the joints can occur due to damage to the central nervous system "trophic center" which serves to regulate bone and joint nutrition. This theory is known as "French theory". Later, "German theory" suggested that changes in the joints occurred as a result of years of accumulation of neglected subclinical trauma due to a low level of joint sensitivity. Neuropathic arthropathy can occur in joints of bedridden patients or those who experienced recurrent joint trauma. In view of these outcomes, there are two fundamental hypotheses that are considered to influence the pathogenesis of neuropathic arthropathy: neurotraumatic hypothesis and neurovascular hypothesis. Neurotraumatic hypothesis recommends that physical muscle reflexes that are typically ensured by joints in scope of movement are diminished or even totally lost with debilitated tactile criticism and diminished proprioception. This causes rehashed mechanical injury (microtrauma) and at last prompts joint obliteration and neuropathic arthropathy. Neurovascular hypothesis recommends that deficiency of neurally animated and controlled vascular reflexes (autosympathectomy) can prompt neuropathic arthropathy bringing about expanded neighborhood blood stream, arteriovenous shunt, erythema, edema, hyperemia, and periarticular bone resorption. Regardless of the absence of proof of the two hypotheses, it is concurred that these two instruments assume a significant part in the pathogenesis of neuropathic arthropathy and that neurovascular systems assume an early part in the pathogenesis of neuropathic arthropathy, trailed by neurotraumatic hypothesis (Nacir et al. 2010).

The objective of the board for neuropathic arthropathy is to treat basic sickness and to decrease deformation rate as low as could really be expected. Treatment of neuropathic arthropathy is generally moderate. Joint goal and supporting are urgent in forestalling conceivable tendon laxity. Non-steroidal mitigating medications might be regulated to treat synovial irritation. Our patient was instructed on the significance of 
joint assurance. Arthrodesis can be performed, however the outcomes may not generally be palatable, in this manner it isn't suggested. Arthroplasty is contraindicated for this situation (Nacir et al. 2010; Yanık et al. 2004). Surgery for the right patient can give a satisfactory outcome; however, it is not the first choice of therapy. Functional orthrosis and physical therapy are priority therapies(Nacir et al. 2010).

\section{Conclusion}

Neuropathic arthropathy after diagnosis and surgery for syringomyelia due to type I Arnold-Chiari malformation should be considered, especially in cases of joint destruction in the absence of pain.

\section{References}

Al-Holou, Wajd N., Samuel Terman, Craig Kilburg, Hugh J. L. Garton, Karin M. Muraszko, and Cormac O. Maher. 2013. "Prevalence and Natural History of Arachnoid Cysts in Adults; Clinical Article." Journal of Neurosurgery 118(2) :222-31.

Aly, Abdel Rahman, Sathish Rajasekaran, Haron Obaid, and Barry Bernacki. 2013. "Bilateral Ulnar Neuropathy at the Elbow Secondary to Neuropathic Arthropathy Associated with Syringomyelia." PM and R 5(6):533-38.

Arunkumar, M. J., I. Korah, and M. J. Chandy. 1998. "Dynamic CSF Flow Study in the Pathophysiology of Syringomyelia Associated with Arachnoid Cysts of the Posterior Fossa." British Journal of Neurosurgery 12(1):33-36.

Bauer, Andrew M., Diane M. Mueller, and John J. Oró. 2005. "Arachnoid Cyst Resulting in Tonsillar Herniation and Syringomyelia in a Patient with Achondroplasia. Case Report." Neurosurgical Focus 19(5):1-7.

Choudhury, Priyam, Ashutosh Mohapatra, Amit Kharat, and Vishva Chauhan. 2019. "Neuropathic Arthropathy of the Shoulder Joint Caused by Syringomyelia : Report of a Rare Case." 2-3.

Cincu, Rafael, Amit Agrawal, and Jose Eiras. 2007. "Intracranial Arachnoid Cysts: Current Concepts and Treatment Alternatives." Clinical Neurology and Neurosurgery 109(10):837-43.

Esra Gurkas1, Buket Yucel Altan2, Kivilcim Gucuyener3, Ebru Kolsal3. 2015. "No Title Cerebellopontine Angle Arachnoid Cyst Associated with Mirror Movements." Journal of Pediatric Neurosciences 10(4):371-73.

Galarza, Marcelo, Antonio López López-Guerrero, and Juan F. Martínez-Lage. 2010. "Posterior Fossa Arachnoid Cysts and Cerebellar Tonsillar Descent: Short Review." Neurosurgical Review 33(3):305-14.

Gönül E, Izci Y, Onguru O. 2007. "Arachnoid Cyst of the Cerebellopontine Angle 
Associated with Gliosis of the Eighth Cranial Nerve." Neuroscience. 700-702.

Hayden, Melanie G., Stephen V. Tornabene, Andy Nguyen, Apurva Thekdi, and John F. Alksne. 2007. "Cerebellopontine Angle Cyst Compressing the Vagus Nerve: Case Report." Neurosurgery 60(6).

J F Martínez-Lage 1, M J Almagro, J Ros de San Pedro, A Ruiz-Espejo, M. FelipeMurcia. 2007. "Regression of Syringomyelia and Tonsillar Herniation after Posterior Fossa Arachnoid Cyst Excision. Case Report and Literature Review." Neurocirugia 18:227-3.

Joshi, Vijay, Ashwin Valsangkar, Satish Nivargi, Nitant Vora, Anish Dekhne, and Amit Agrawal. 2013. "Giant Posterior Fossa Arachnoid Cyst Causing Tonsillar Herniation and Cervical Syringomyelia." Journal of Craniovertebral Junction and Spine 4(1):43-45.

Martínez-Lage, Juan F., Antonio Ruiz-Espejo, Encarna Guillén-Navarro, and María José Almagro. 2008. "Posterior Fossa Arachnoid Cyst, Tonsillar Herniation, and Syringomyelia in Trichorhinophalangeal Syndrome Type I: Case Report." Journal of Neurosurgery 109(4):746-50.

Milhorat, Thomas H., Mike W. Chou, Elizabeth M. Trinidad, Roger W. Kula, Menachem Mandell, Chantelle Wolpert, and Marcy C. Speer. 1999. "Chiari I Malformation Redefined: Clinical and Radiographic Findings for 364 Symptomatic Patients." Neurosurgery 44(5):1005-17.

Nacir, Baris, S. Arslan Cebeci, E. Cetinkaya, A. Karagoz, and H. R. Erdem. 2010. "Neuropathic Arthropathy Progressing with Multiple Joint Involvement in the Upper Extremity Due to Syringomyelia and Type I Arnold-Chiari Malformation." Rheumatology International 30(7):979-83.

Oldfield, E. H., K. Muraszko, T. H. Shawker, and N. J. Patronas. 1994. "Pathophysiology of Syringomyelia Associated with Chiari I Malformation of the Cerebellar Tonsils. Implications for Diagnosis and Treatment." Journal of Neurosurgery 80(1):3-15.

Ramesh S, Raju S. 2015. "Suprasellar Arachnoid Cyst Presenting with BobbleHead Doll Syndrome: Report of Three Cases." J Pediatr Neurosci. 10:18-21.

Yanık, Burcu, Safiye Tuncer, and Bülent Seçkin. 2004. "Neuropathic Arthropathy Caused by Arnold-Chiari Malformation with Syringomyelia." Rheumatology International 24(4):238-41. 